\title{
WAVELET ANALYSIS FOR INVESTIGATION OF PRECISE GNSS SOLUTIONS' CREDIBILITY
}

\author{
Janusz Bogusz, Anna Klos \\ Centre of Applied Geomatics, Military University of Technology, Warsaw \\ e-mail: jbogusz@wat.edu.pl
}

\begin{abstract}
This publication presents the results of searching short-term oscillations of the ASG network sites using wavelet transform. Polish Active Geodetic Network (ASG-EUPOS) is the multifunctional precise satellite positioning system established by the Head Office of Geodesy and Cartography in 2008. The adjusted network consisted of over 130 stations from Poland and neighbouring countries. The period covered observations gathered from June 2008 to July 2010. The method of processing elaborated in the CAG (Centre of Applied Geomatics, Warsaw Military University of Technology), which is one of the 17 EPN LAC (EUREF Permanent Network Local Analysis Centre) acting now in Europe, established at the end of 2009, is similar with the official one used in EPN. It is based on the Bernese 5.0 software, but the difference to the EPN's solutions lies in the resolution of resulting coordinates. In the presented research the 1-hour sampling rate with 3-hour windowing (66\% of correlation) is applied. This allows us to make the interpretations concerning short period information in GNSS (Global Navigation Satellite System) coordinates series. Analyses using FFT and least squares (tidal) gave very coherent results and confirmed several millimetres diurnal and sub-diurnal oscillations. Wavelet analysis is aimed at the investigation of credibility of the precise GNSS solutions in terms of changes of the amplitude of oscillations in time. As a result of this study the changes in the amplitude of oscillations at diurnal and sub-diurnal frequency bands were obtained. These could be caused by the artificial modulations of the near-by frequencies, but also some geophysical signals could be clearly distinguished. Additionally the comparison of Continuous Wavelet Transforms of near stations (three pairs from ASG-EUPOS network) was performed. This comparison showed different behaviour of oscillations of residual coordinates, mainly due to the different thermal response or artefacts related to the multipath effects or influences of the network geometry.
\end{abstract}

Keywords: GNSS, wavelet transform, short-period coordinates changes.

\section{INTRODUCTION}

The Fourier Transform (FT) depends on going from the field of time to the field of frequency and separates the given time series on the sinusoids of different frequencies. In spite of the fact that it is used nowadays in more and more cases, it has also some disadvantages. The FT of time series loses information about changes of the amplitude in time. Moreover FT gives very reliable results only when using it on stationary signals. In case of geodetic time series the signals have no stationary characteristics, then FT gives unreliable results.

A continuous wavelet transform (CWT) is used to divide a continuous-time function into wavelets. Unlike Fourier transform, this transform possesses the ability to construct a timefrequency representation of a signal that offers very good time and frequency localization, however they cannot be improved simultaneously. 
The merits of its application to study changes associated with the Earth's gravity field has been previously demonstrated e.g. in the paper by Lintao et al., 2000 or Sun et al., 2008 and for changes in the levels of the oceans in the publication by Flinchema and Jay, 2002. In Poland, this problem has addressed in the publications by Araszkiewicz and Bogusz, 2008, 2010 for the processing gravimetric observations - Earth tides made in the Observatory in Jozefoslaw by LC\&R model ET No. 26 gravimeter. The determination of diurnal, semidiurnal and 8-hours (consecutive harmonics) oscillations and their changes over time by using a complex Morlet wavelet has been possible.

Analysis of the outcomes from advanced GNSS (Global Navigation Satellite System) data processing on ASG-EUPOS (Polish National GBAS - Ground Base Augmentation System) using FFT were previously discussed in the papers by Araszkiewicz et al., 2009, 2010; Bogusz and Figurski, 2010. These researches showed the short-period residual oscillations in the geodetic coordinates at the level of several millimetres. Taking into account that ASGEUPOS in the near future begins to play the role of national basic reference network these changes seem to be significant. In that case the wavelet transform appears to be the best method to investigate the time-frequency properties of the GNNS solutions.

\section{CONTINUOUS WAVELET TRANSFORM}

The Fourier transform, in essence, decomposes a function into sinusoids of different frequencies which can sum to the original waveform. This transform provides a frequency representation of the signal i.e., gives information which frequency components exist in the signal. The Fourier transform is an excellent tool for the analysis of the stationary signals in which frequencies components exist all time.

D. Gabor adapted the Fourier transform to analyze only short segments of a signal. These small sections are assumed to be stationary and can be transformed separately using a window function, which is moved along the signal. Gabor's adaptation, called the windowed Fourier transform (WFT), maps a signal into a two-dimensional function of time and frequency. The windowed Fourier transform provides accurate information about the signal simultaneously in the time domain and in the frequency domain (Fig. 1). However, all functions, including window functions, obey the Heisenberg uncertainty principle, which states that the sharp localization in time and in frequency are mutually exclusive i.e. it is impossible to find out what spectral component exist at what instances of time. Only the time intervals in which certain hand of frequencies exists can be known.

In the case of non stationary signals, the ultimate solution is the wavelet transform which provides time-scale representation of the signal. The wavelets are the new way to see and represent a signal, but they are alternatives to the Fourier transform rather than its replacement. But in contrast to the Fourier transform which during transformation to the frequency domain loses time information, wavelets have scale and time aspects as well. Wavelet analysis is capable of revealing aspects of data that other signal analysis techniques miss, aspects like trends, breakdown points, discontinuities in higher derivatives, and selfsimilarity. Furthermore, because it affords a different view of data than those presented by traditional techniques, wavelet analysis can often compress or de-noise a signal without appreciable degradation. The changing signals needed although much more elastic approach, where the size of the window can be changed depends on what information (in time or frequency domain) we want to obtain. Recently the representations of the signals as timefrequencies are the most useful in spectral analysis and converting the non stationary signals. The two types of wavelet transform are marked out: the discrete wavelet transform (DWT) for discrete signals and a continuous one (CWT) for continuous signals. The wavelet analysis is the sort of window technique with the changing window size. 
It represents the next logical step after WFT: a windowing technique with variable-sized regions and allows the use of long time intervals while obtaining more precise low-frequency information, and shorter regions when high-frequency information is wanted (Fig. 1).
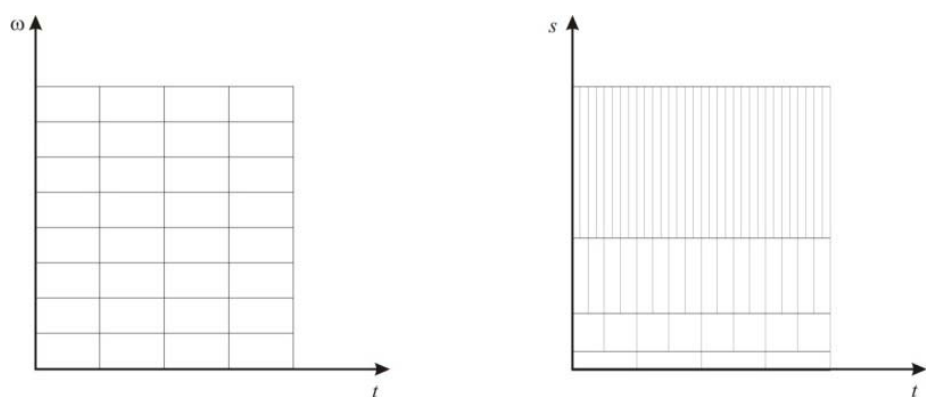

Fig. 1. WFT in the time-frequency plane (left) and CWT in the time-scale plan (right)

The wavelets are the "small waves" that are made from a one basic wave, called "a mother wavelet" by scaling and shifting it. The wavelet analysis depends on sharing the signal on smaller parts and compare them with shifted and scaled "children" of "mother wavelet". The most important thing about wavelets is their lack of symmetry and dying down with passing the time. In wavelet analysis the use of a fully scalable modulated window solves the signal-cutting problem. The window is shifted along the signal and for every position the spectrum is calculated. Then this process is repeated many times with a slightly shorter (or longer) window for every new cycle. At the end the result will be a collection of time-frequency representations of the signal, all with different resolutions. Because of it we can speak of a multiresolution analysis (Box and Jenkins, 1970).

The continuous wavelet transform is defined as the sum over all time of the signal multiplied by scaled and shifted versions of the wavelet function $\psi$ (Box and Jenkins, 1970):

$$
C(\text { scale }, \text { position })=\int f(t) \cdot \psi(\text { scale }, \text { position }, t) d t
$$

CWT of a signal is a sequence of projections onto rescaled and translated versions of analysing functions of wavelets (Mallat, 1999):

$$
C W T_{x}^{\psi}(s, \tau)=\int_{-\infty}^{\infty} x(t) \psi_{s, \tau}^{*}(t) d t
$$

where:

$$
\psi_{s, \tau}(t)=\frac{1}{\sqrt{|s|}} \psi\left(\frac{t-\tau}{s}\right), \quad s, \tau \in R, \quad s \neq 0
$$

The above equation presents a wavelet function, which depends on two parameters: $s-$ scale coefficient and $t$ - time shift.

In this study the complex Morlet wavelet was used (Goupillaud et al., 1984):

$$
\psi(x)=\frac{1}{\sqrt{\pi \cdot f_{b}}} e^{2 i \pi f_{c} x} e^{-\frac{x^{2}}{f_{b}}}
$$

which is the function of two parameters: $f_{b}$ - bandwidth parameter and $f_{c}$ - center frequency. 
Wavelet analysis does not use a time-frequency region, but rather a time-scale region. Wavelets allow using of short time intervals to obtain precise high frequency information and using longer time intervals for low frequency information. A width and height of the box change but the area is constant. Each box represents equal portion of the time-frequency plane with different proportions to time and frequency (Fig. 1). A way to relate scale to frequency is the computation of the centre frequency $f_{c}$ of the wavelet and use the following relationship (Misiti et al., 2000):

$$
f_{a}=\frac{f_{c}}{a \cdot T}
$$

where $a$ is a scale factor, $T$ is the sampling period, $f_{c}$ is the centre frequency of a wavelet in $\mathrm{Hz}$, and $f_{a}$ is the pseudo-frequency corresponding to the scale $a$.

\section{DATA AND PROCESSING}

This research was realized upon the data concerning hourly changes of the coordinates from the ASG-EUPOS. The observation data are RINEX files, available in the interval of 30 seconds as daily files. The observations hold almost 90 Polish and neighbouring countries (Germany, Czech Republic, Slovakia, Ukraine, Belorussia, and Lithuania) sites with GPS module. 14 Polish sites is equipped with GPS/GLONASS module. 5 stations were assumed as referenced in the processing (ONSA, METS, POTS, BOR1 and WTZR), 24 of them are simultaneously the EPN sites (Fig. 2). The processing of the observations from ASG-EUPOS has been done in Bernese 5.0 software (Dach et al., 2007). The available data cover period from 8.06.2008 to 18.06.2010. Data processing strategy as well as the applied models were broadly described in the paper by Araszkiewicz et al., 2010.

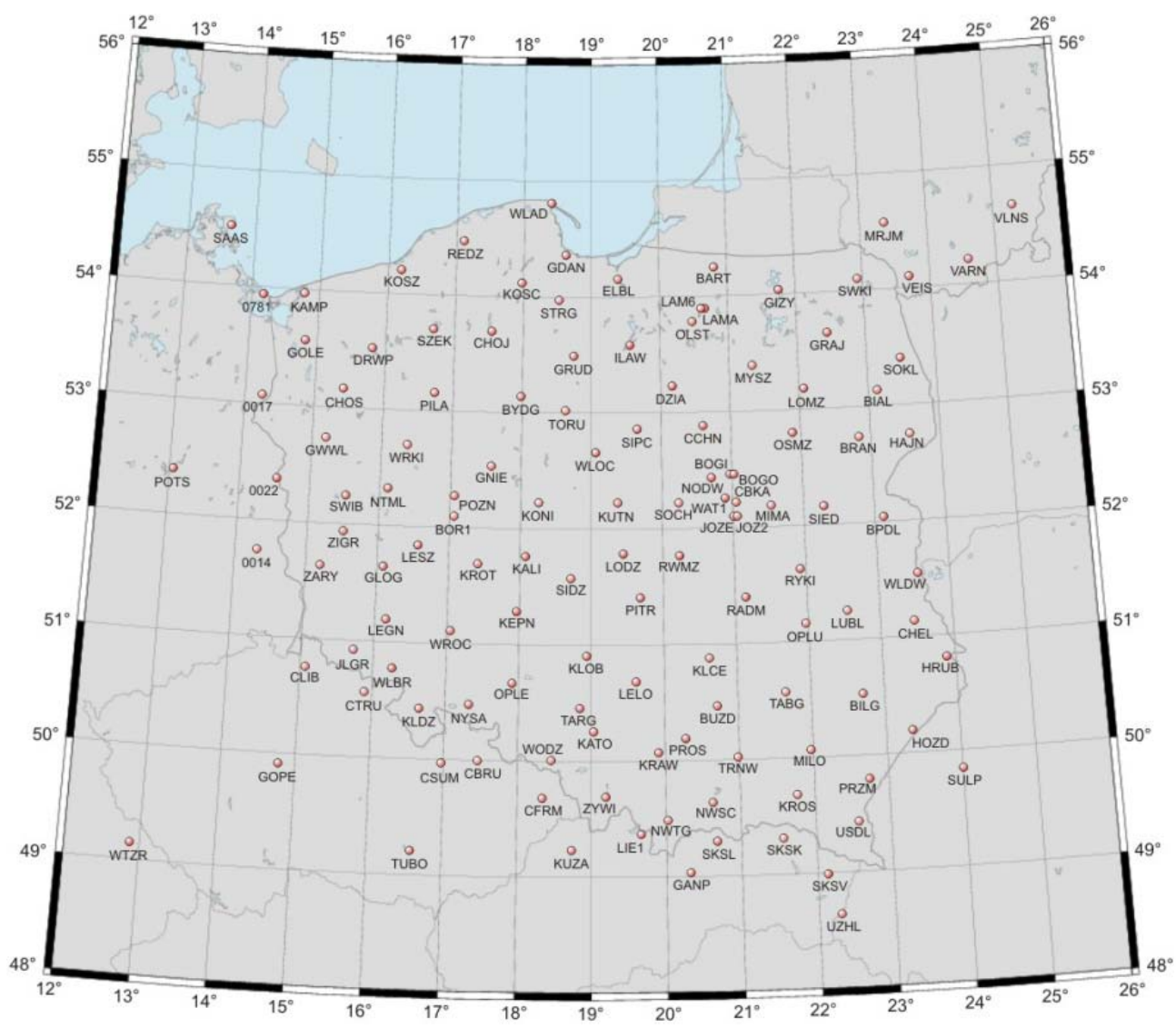

Fig. 2. Map of GNSS sites 
The hourly solutions conduce to the monitoring of stations' coordinates validity. The evaluation of solutions' stability in the short periods and observations control as the great material for later analysis and interpretations also of geodynamic character. The XYZ coordinates in ITRF2005 reference frame, taken from ASG files, were re-calculated to topocentric coordinates NEU (North, East, Up) since orthocartesian XYZ coordinates are not suitable to interpret the geodynamical and geophysical phenomena.

\section{RESULTS}

Continuous wavelet transform allowed to obtain information about the nature of the variability of the short-term oscillations in time. The whole project was realized in MATLAB ${ }^{\circledR}$ (license number \#350334) while it gives a broad range of wavelets to use and once written algorithm can be used for more than one file. The continuous Morlet wavelet with the values $f_{b}=25$ and $f_{c}=5$ was used. This wavelet exactly reproduces the changes with characteristic amplifications for signals and does not fade the data.

As the result of the written algorithm three graphs for each of the ASG station were obtained on which we can distinctly see the expected periods with 24-, 12-, 8-hour changes, when did their changes took place and how high they were. The exemplary transforms for Polish station Goleniow are presented in Fig. 3. The similar graphs were obtained for the rest of the adjusted stations.
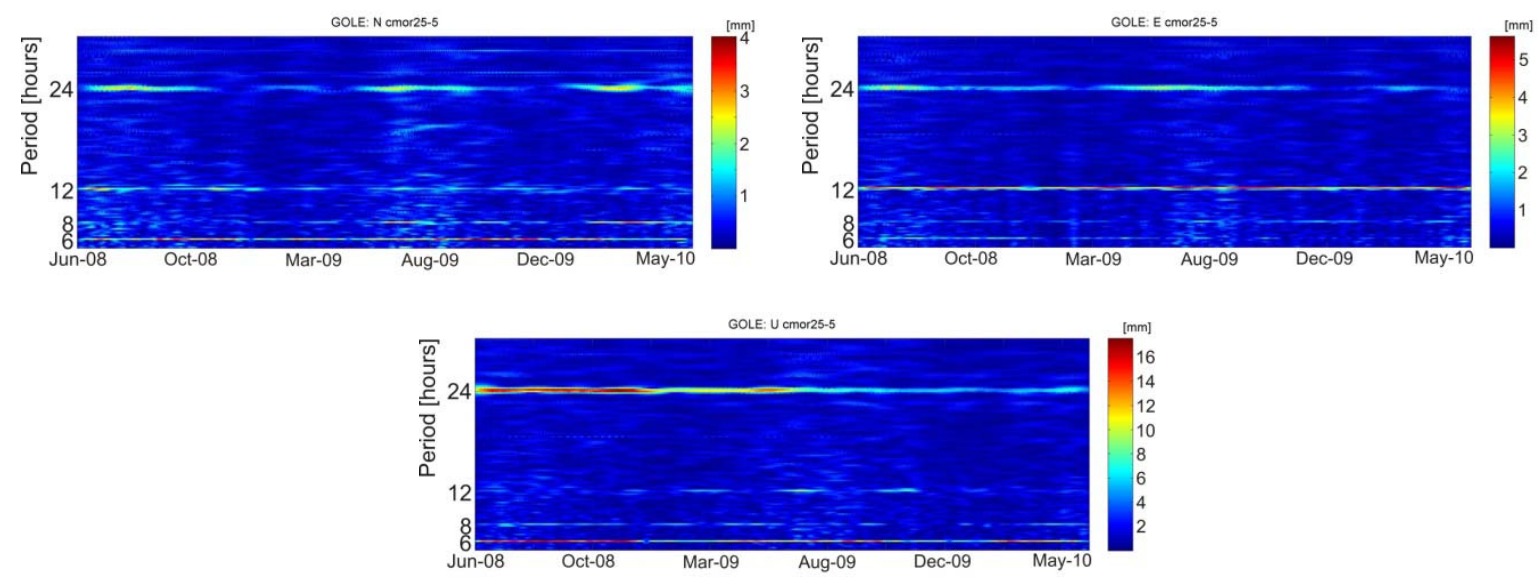

Fig. 3. CWT transform using Morlet wavelet for GOLE site (N, E, U from up)

The wavelet analysis is capable to reveal aspects of data that other signal analysis techniques miss, aspects like trends, breakdown points, discontinuities in higher derivatives, and self-similarity. Fig. 4. shows the abnormal behaviour of the topocentric coordinates in the East-West direction of Biala Podlaska site, caused probably by the deficiencies in ionosphere and troposphere modelling, effects on residual errors in satellites' orbiting or multipath effect. The smudge in the turn of December and January is unexpected. Coordinate changes are reflected in the transform graph. In Fig. 4. many of picks are much higher than the mean value is, therefore the graph in CWT is so coloured in this period. Beyond above smudge, there are many vertical changes in colour on the CWT graph. In much cases the light colour which indicates outliers is seen. To avoid such a strong artificial changes, the filtration on the $\mathrm{N}, \mathrm{E}, \mathrm{U}$ time series using $3 \sigma$ criterion was imposed. 

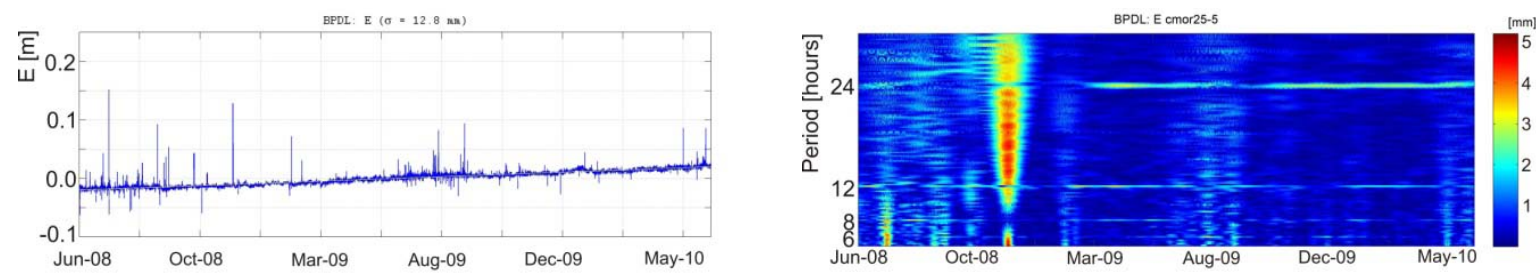

Fig. 4. Changes of E component of BPDL site (left) and its CWT

These smudges indicate the gaps or the high local fluctuation in coordinates. For example on the all of the analysed graphs (CWTs) the explicit intermittences in the time from May to September 2009 can be seen (as shown on example in Fig. 4). It can testify to some changes of geophysical origin during this time.

Moreover few of the ASG stations are collocated, they lie in the local ties, in the distance of few meters. This chapter would like to be treated as a voice in the discussion on the differences of GNSS networks stabilization points. Antennas on the left side of figures 5-7 are placed on pillars, right on the roofs. BOGO and BOGI sites (Fig. 5) have very similar characteristics (related to the $U p$ component), the prevalence of a diurnal variation with almost the same amplitude, only we can see clearer BOGO 8-hour oscillation. Almost no intermittences on both sites could be noticed. LAMA site seems to be much more stable than LAM6 (Fig. 6), but it has different characteristics(two times bigger amplitude), with the predominant semidiurnal oscillation. The intermittences in these stations behave in the similar way. JOZE seems to be much more unstable point (the maximum amplitude of three times bigger) than JOZ2 (Fig. 7), but much more intermittences is visible on JOZ2. The curious thing about them is their form - some of them are short in period and have a form of funnel while the others are much more overstretched and have a form of rectangle.
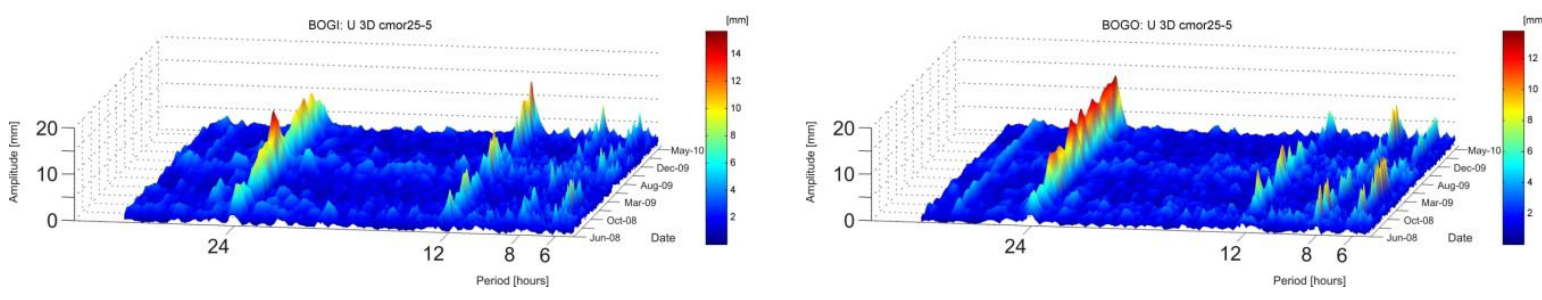

Fig. 5. CWTs (Up component $[\mathrm{mm}]$ ) of near-by sites BOGO (left) and BOGI (right)
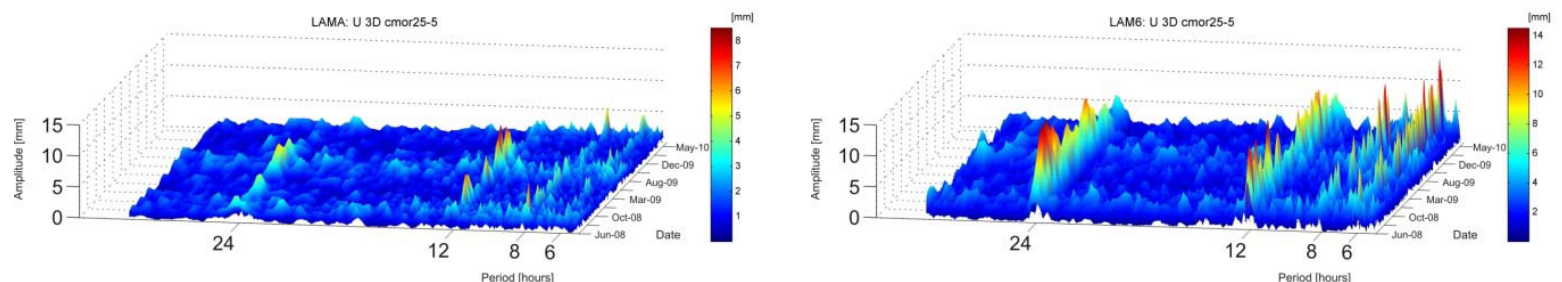

Fig. 6. CWTs (Up component [mm]) of near-by sites LAMA (left) and LAM6 (right)
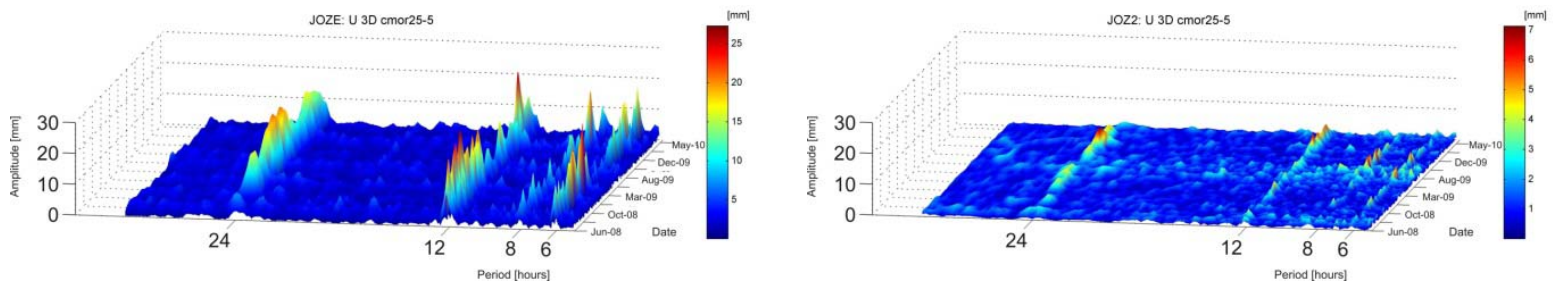

Fig. 7. CWTs (Up component [mm]) of near-by sites JOZE (left) and JOZ2 (right) 
Generally no dependences between the antennas' mounting were noticed. The coordinates (by means of short-term oscillations and their changes) behave in irregular way. Although some geophysical effects (for example that in the December 2009) appear not only on the near-by sites but on the all ASG points. The oscillations in this period are very small and the curve turns to form of the funnel. It might be some geodynamical effect due to the fact that it does not repeat in the further time (Fig. 10).

Since the wavelets reveal changes of the amplitude's oscillation in time, the horizontal sections in 24-, 12- and 8- hourly changes were made (the selected raw of the CWT matrix). Fig. 8 shows changes of amplitude of U component's oscillation in time. In all 24-hourly graphs the changes have greater amplitude than for other periods, moreover these graphs are much more smooth. The changes are not as rapid in time, as they are in 12-, and 8-hours. Some reproducibility in 12-hour changes can be seen (one month period). 8-hourly graphs are the most torn - the coordinates change is the rapidest in time.
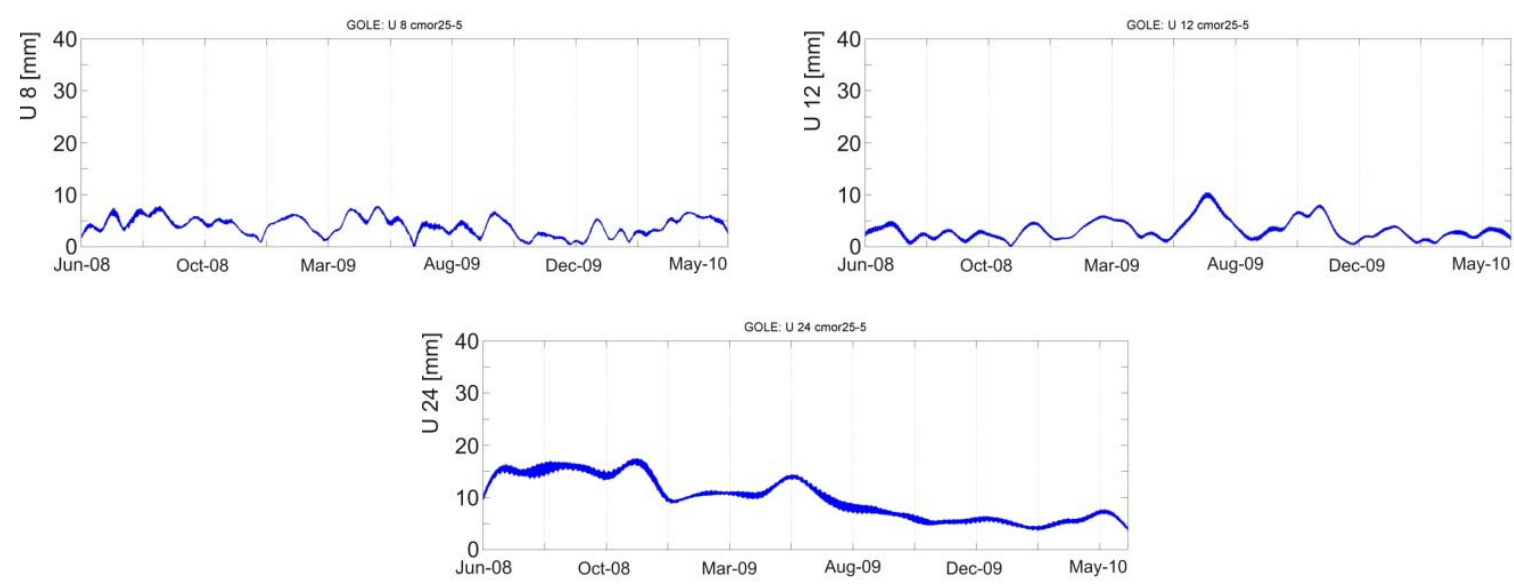

Fig. 8. Horizontal sections for $U$ for GOLE station (24, 12 and 8 hours)

Analysing of these section lead us also to the unpredicted behaviour of some ASG sites. Fig. 9 shows the growth in the 12-hours amplitude's oscillations in January 2010 for Klobuck site. This growth was probably caused by environmental effects on the station. In that time the large snowfalls took place in Poland which made the station to be unstable. These changes were also noticed at other sites of the network like Jozefoslaw (Fig. 10) or Lelow (Fig. 11).
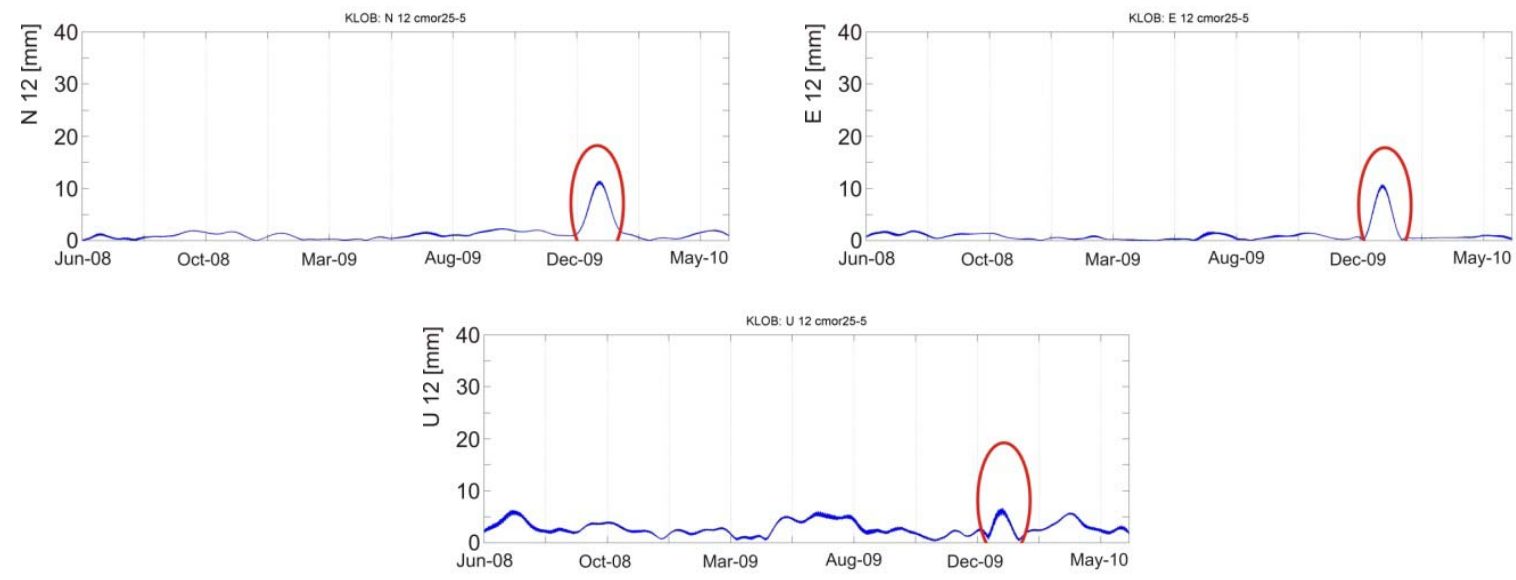

Fig. 9. 12-hour sections for KLOB station 

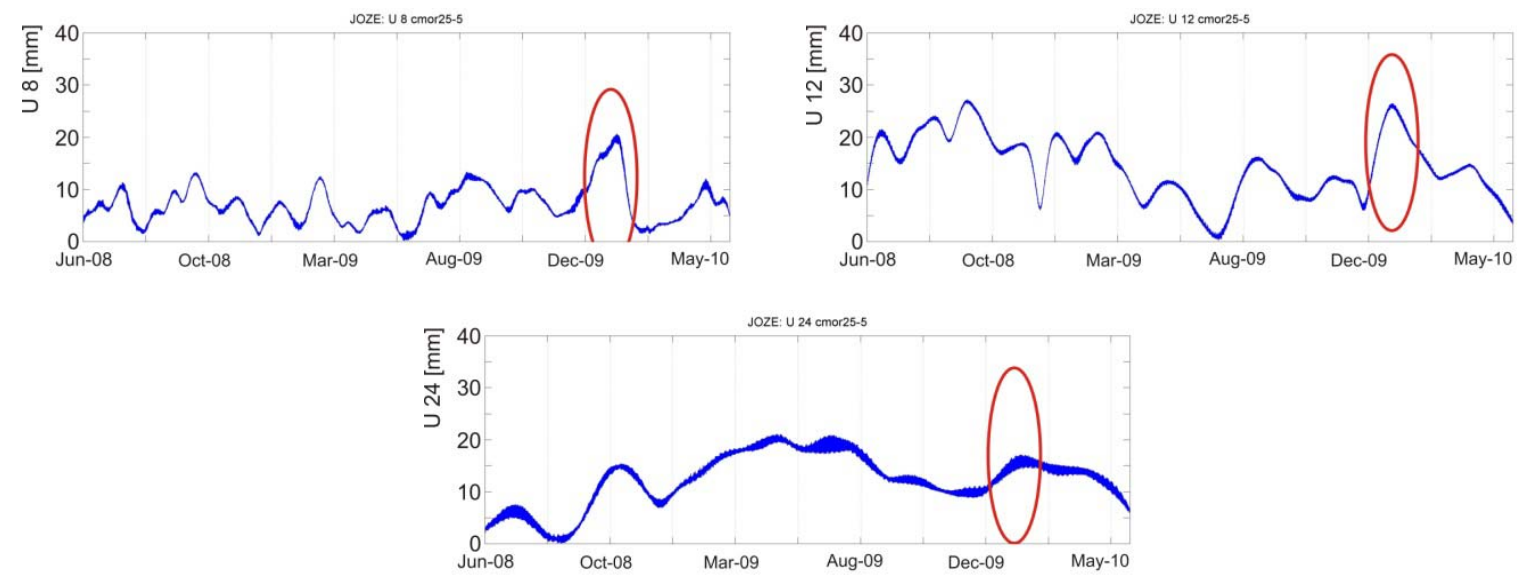

Fig. 10. Horizontal sections for $U$ for JOZE station $(24,12$ and 8 hours)
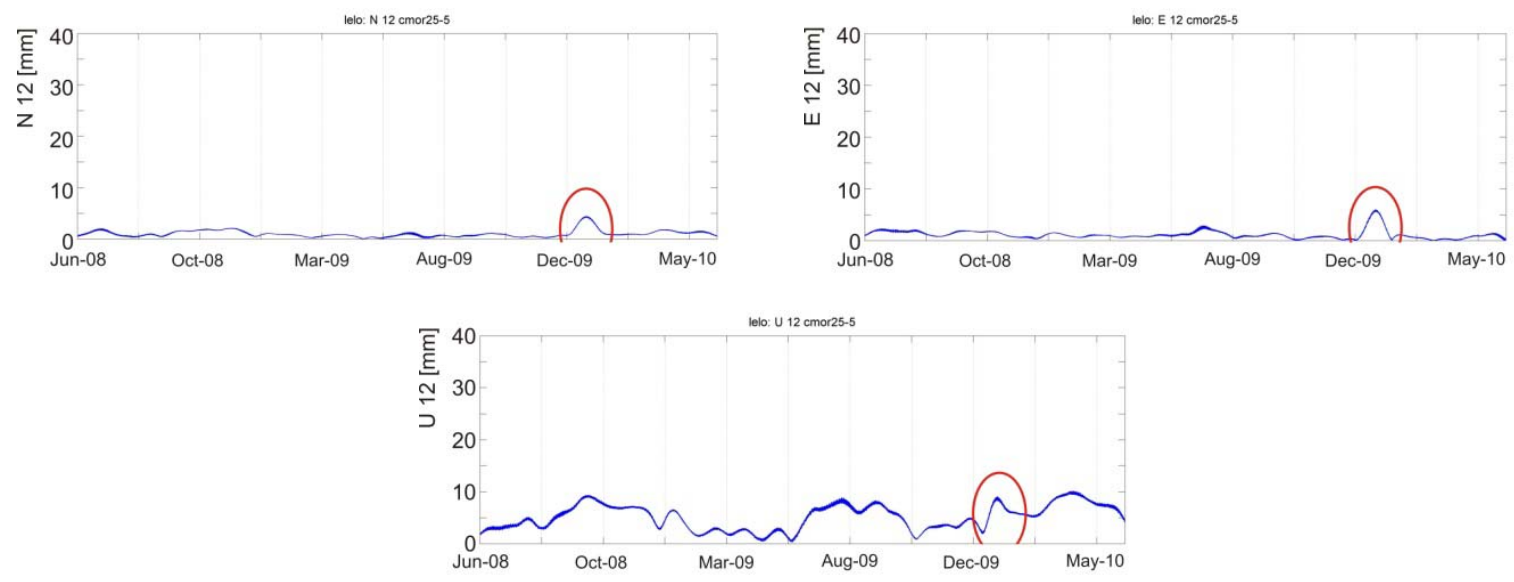

Fig. 11. Horizontal sections for $U$ for LELO station $(24,12$ and 8 hours $)$

Other local effects are reflected in the Wlodawa (WLDW) site's CWT. This site has the most significant changes of the amplitude's oscillations in time. It lies very close to the Bug river pool, so the local hydrology could be dominant in the residua of coordinates (the maximum could be clearly seen during the summer-time). $40 \mathrm{~mm} \mathrm{12-hour} \mathrm{oscillations} \mathrm{in} \mathrm{U}$ component cause the station to be very unstable in short period (Fig. 12).
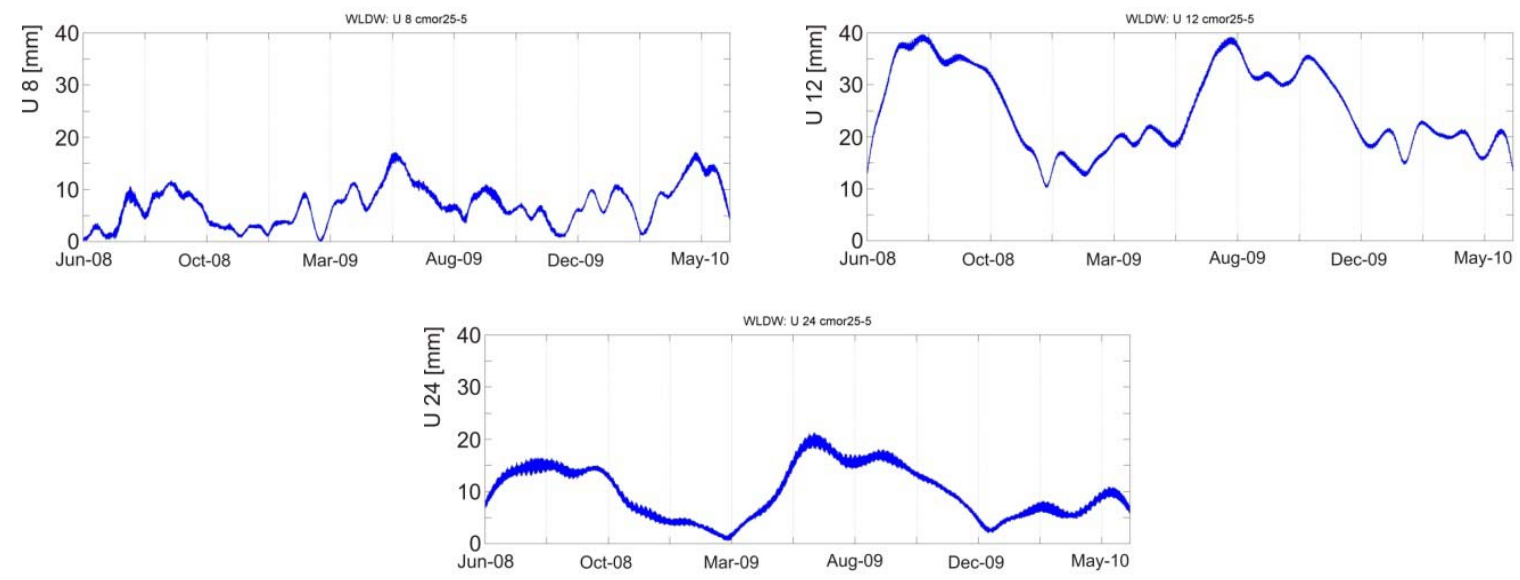

Fig. 12. Horizontal sections for U for WLDW station (24, 12 and 8 hours) 
The authors do not have data on changes of the river's water level, but the impact of the local hydrology influence on the site's position is rather certain (Fig. 13).

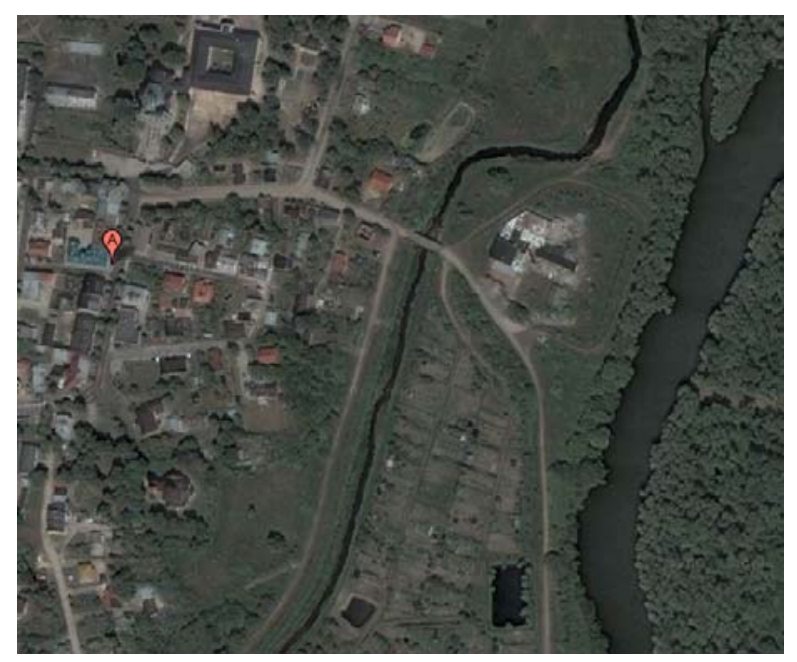

Fig. 13. Location of WLDW station (marked "A") in the river pool (Google Earth)

The main advantage of using wavelet transform is that this method allows to distinguish stable sites and the unstable ones (Fig. 14). This distinction is very important due to the fact that ASG-EUPOS in the near future begins to play the role of national basic reference network.
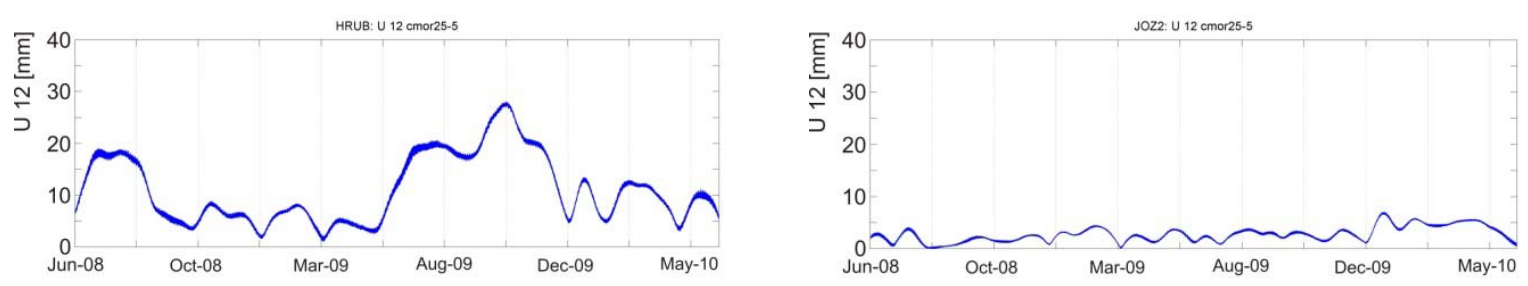

Fig. 14. Example of the less (HRUB - left) and more stable (JOZ2 - right) site of ASG network

\section{DISCUSSION ON THE RESULTS}

1. The main advantage of using wavelet transform is that this method allows to distinguish stable sites and the unstable ones. This distinction is very important due to the fact that ASG-EUPOS in the near future begins to play the role of national basic reference network.

2. All wavelets approachable in MATLAB ${ }^{\circledR}$ has been tested: beginning from Daubechies, ending on Mexican Hat. The best for this type of data is continuous Morlet wavelet called "cmor 25-5" (the values $f_{b}=25$ and $f_{c}=5$ were used). This wavelet exactly reproduces the changes with characteristic amplifications for signals and does not fade the data.

3. The intermittences of the signal have been discovered. The filtration of the data helped to cancel the high changes, but the smaller still left. These could be caused by some geophysical changes. The intermittences are much more visible in the period of May and September than in others - some geophysical changes took place then. Moreover the same changes can be seen in the CWT graphs for the near-by stations of ASG. 
The wavelet's parameters were chosen empiric by the fact of how the spectrogram looked like. By the use of "cmor25-5" the changes of amplitude in time can be interpreted the best. The decreasing of the bandwidth parameter and the centre frequency of a wavelet makes the spectrogram too diffused, it cannot be found out on the period axis when exactly the change took place (Fig. 15), on the other hand increasing them makes the oscillations almost invisible (Fig. 16).
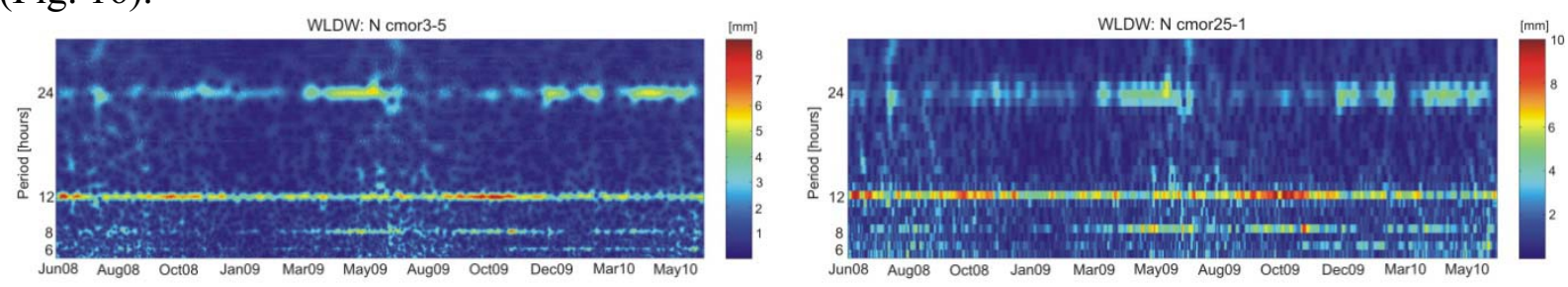

Fig. 15. Spectrograms for "cmor3-5" (left) and "cmor25-1" (right)
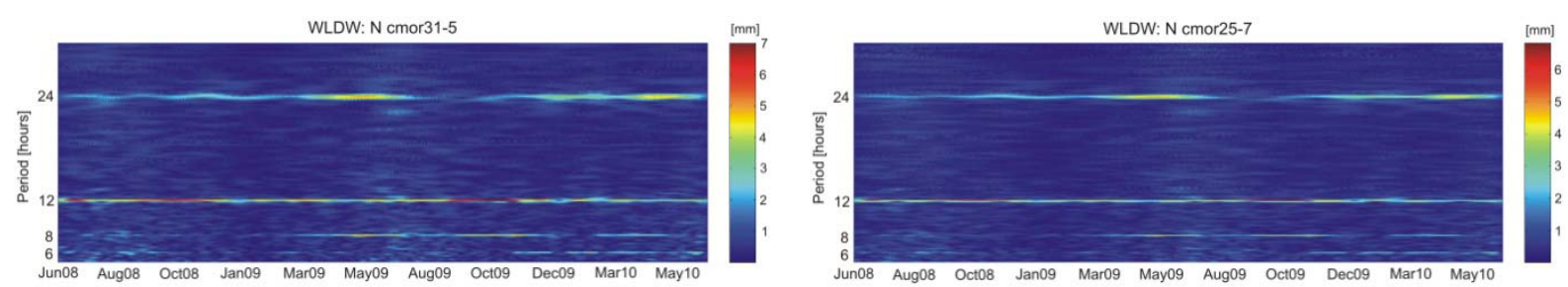

Fig. 16. Spectrograms for "cmor31-5" (left) and "cmor25-7" (right)

4. Still to be done: for complete elimination of artificial oscillation analysis of artificial oscillations there should be developed a method to eliminate them based on the complex decomposition method, for determination of the transfer of the energy between frequencies cepstral analysis should be made.

\section{ACKNOWLEDGMENTS}

Dr. Mariusz Figurski (CAG MUT) for his commitment to the GNSS data processing and Dr. Wieslaw Kosek (SRC PAS) for very efficient discussions are gratefully acknowledged.

\section{REFERENCES}

Araszkiewicz A., Bogusz J. (2010): "Application of wavelet technique to the Earth tides observations analyses". Marees Terrestres Bulletin d'Informations (BIM), No 146, ISSN No 0542-6766, 2010, pp. 11789-11798.

Araszkiewicz A., Bogusz J., Figurski M. (2009): "Investigation on tidal components in GPS coordinates". Artificial Satellites, Volume 44, Number 2 / 2009, DOI 10.2478/v10018009-0020-9, pp. 67-74.

Araszkiewicz A., Bogusz J., Figurski M., Szafranek K. (2010): “Application of short-time GNSS solutions to geodynamical studies - preliminary results". Acta Geodynamica et Geomaterialia, Vol. 7, No. 3 (159), 2010, pp. 295-302.

Bogusz J., Figurski M. (2010): "Short-period information in GPS time series". Artificial Satellites, Vol. 45, Number 3 / 2010.

Box G., Jenkins G. (1970): "Time series analysis: Forecasting and control”. San Francisco: Holden-Day, 1970. 
Rolf D., Hugentobler U., Fridez P., Meindl M. (Editors) (2007): "Bernese GPS Software Version 5.0". Astronomical Institute, University of Bern, 2007.

Flinchema E. P., Jay D. A. (2002): “An Introduction to Wavelet Transform Tidal Analysis Methods". Estuarine, Coastal and Shelf Science, Volume 51, Issue 2, August 2000, pp. 177-200.

Goupillaud P., Grossmann A., Morlet J. (1984): “Cycle-octave and related transforms in seismic signal analysis". Geoexploration, 23, pp. 85-102.

Lintao L., Houze X., Heping S., Xinghua H. (2000): "Wavelet approach to the determination of gravity tide parameters". Science in China Series D: Earth Sciences, Volume 43, Number 2, DOI: 10.1007/BF02878145, pp. 158-165.

Mallat S. (1999): “A wavelet tour of signal processing, 2nd Edition”. Academic Press, New York, 1999.

Melchior P. (1983): “The Tides of the Planet Earth”. Pergamon Press, 1983.

Misiti M., Misiti Y., Oppenheim G., Poggi J.-M. (2000): “Wavelet toolbox". The MathWorks Inc.

Rucka M., Wilde K. (2007): "Application of wavelet analysis in damage detection and localization”. Wydawnictwo Politechniki Gdańskiej, Gdańsk 2007.

Sun H. P., Zheng W. D., Xu J. Q, Hsu H. Z (2008): "Detection of the Special Gravity Signals in Sub-tidal Band by Using a Wavelet Techniques". Presented at the Workshop on Analysis of Data from Superconducting Gravimeters and Deformation Observations Regarding Geodynamic Signals and Environmental Influences, Jena, March 27 - 31, 2006.

Tamura, Y. (1987): “A harmonic development of the tide-generating potential”. Bull. Info. Maries Terrestres, Vol. 99, pp. 6813-6855.

Received: 2011-01-18,

Reviewed: 2011-03-02, by W. Rohm,

Accepted: 2011-03-14 